\title{
Facial Subcutaneous Emphysema As A Rare Complication Of Tonsillectomy
}

Mark Paul ${ }^{1}$ (MBBS), Najihah Hanim Asmi ${ }^{1}$ (MS ORL-HNS), Rohaida Ibrahim ${ }^{1}$ (M.Med ORL-HNS), Eshamsol Kamar Omar $^{1}$ (M.Med ORL-HNS), Irfan Mohamad ${ }^{2}$ (M.Med ORL-HNS)

${ }^{1}$ Department of Otorhinolaryngology, Hospital Sultan Haji Ahmad Shah, Temerloh, Pahang.

${ }^{2}$ Department of Otorhinolaryngology-Head \& Neck Surgery, School of Medical Sciences, Universiti Sains Malaysia Health Campus, Kota Bharu, Kelantan.

\section{ABSTRACT}

Tonsillectomy is a very common procedure in Otolaryngology practice. Common complications include bleeding, which can be primary or secondary due to infection of the tonsillar bed. Subcutaneous emphysema after a tonsillectomy is very rare. We report a 19-year-old girl who developed cervicofacial subcutaneous emphysema several hours after tonsillectomy with successful conservative treatment outcome.

KEYWORDS: Tonsillectomy; emphysema; face

\section{INTRODUCTION}

Tonsillectomy is one of the most commonly performed surgeries in Otolaryngology practice. Post tonsillectomy haemorrhage tops the list of post tonsillectomy complications. Subcutaneous emphysema, and pneumomedastinum after a tonsillectomy is an extremely rare complication. It can be fatal if severe. Only about 30 of such cases were reported, that made the incidence being less than $1 \%{ }^{1}$. Fortunately, most of these cases resolved spontaneously ${ }^{1-3}$. Various possible mechanisms of the complication were implicated. One of them was tonsillectomy caused a breach in the pharyngeal mucosa and may injure the underlying muscles, contributing to the development of subcutaneous emphysema ${ }^{4}$.

\section{CASE REPORT}

A 19-year-old girl, with frequent episodes of tonsillitis was referred for an elective tonsillectomy. Clinical examination showed bilateral

Corresponding author:

Assoc. Prof. Dr. Irfan Mohamad, Department of Otorhinolaryngology-Head \& Neck

Surgery,

School of Medical Sciences,

Universiti Sains Malaysia Health Campus, 16150 Kota Bharu, Kelantan.

Tel: $609-7676420$

Fax: 609-7676424

Email:irfankb@usm.my enlarged tonsils grade 3 without adenoid hypertrophy. She underwent tonsillectomy under general anesthesia. The tonsils were dissected using monopolar diathermy, while bipolar diathermy was used to secure the bleeding. Lignocaine gel was applied onto the tonsilar bed for local anaesthetic effect.

Intraoperative findings showed the grade 3 enlarged tonsils. The plane of tonsils were well-demarcated, dissection was unremarkable and bleeding were minimal intraoperatively. Intubation, ventilation and extubation by anaesthetist were smooth. There was no strenuous coughing or vomiting after extubation. She was observed for one hour at the Post Anaesthesia Care Unit (PACU) and was returned to the general ward uneventfully.

At 3 hours, post surgery, she started to develop swelling over the left cheek extending down to the neck (Figure 1). The swelling was not painful, warm or tense. Skin over the swelling was normal and there was crepitus noted upon palpation indicating the presence of subcutaneous emphysema. She was observed and managed conservatively with supplemental oxygen via nasal prong and kept fasted. On the next day after surgery, the subcutaneous emphysema extended from the left side of her face to the left side of her neck and upper part of her chest. The airway was not compromised and there was no problem with deglutition. 


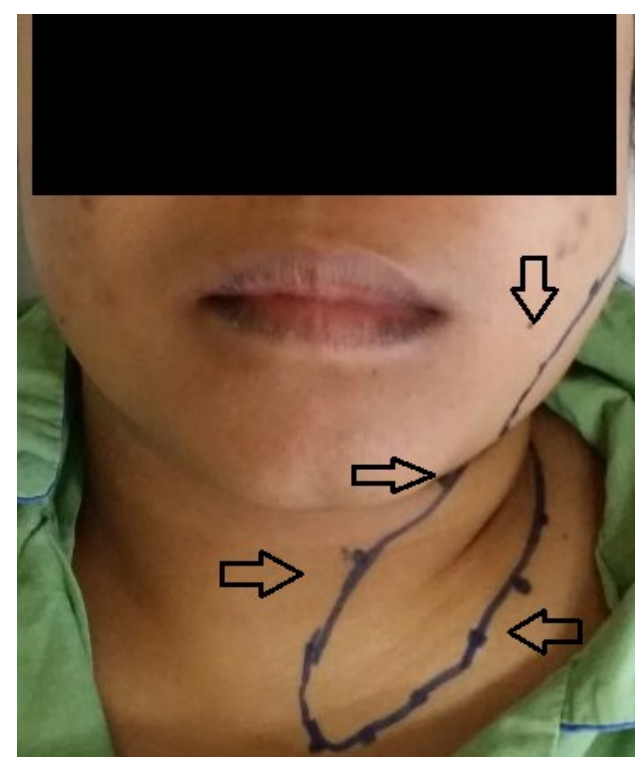

Figure 1A: Subcutaneous emphysema on the left cheek and neck (Anterior view)

Radiological examinations confirmed the presence of the subcutaneous emphysema in the neck (Figure 2 \& 3). There was no evidence of pneumothorax or pneumomediastinum seen.

We commenced her on intravenous amoxicillinclavulanic acid and closely monitored her clinical and vital signs in the general ward.

On the 4th day after surgery, her subcutaneous

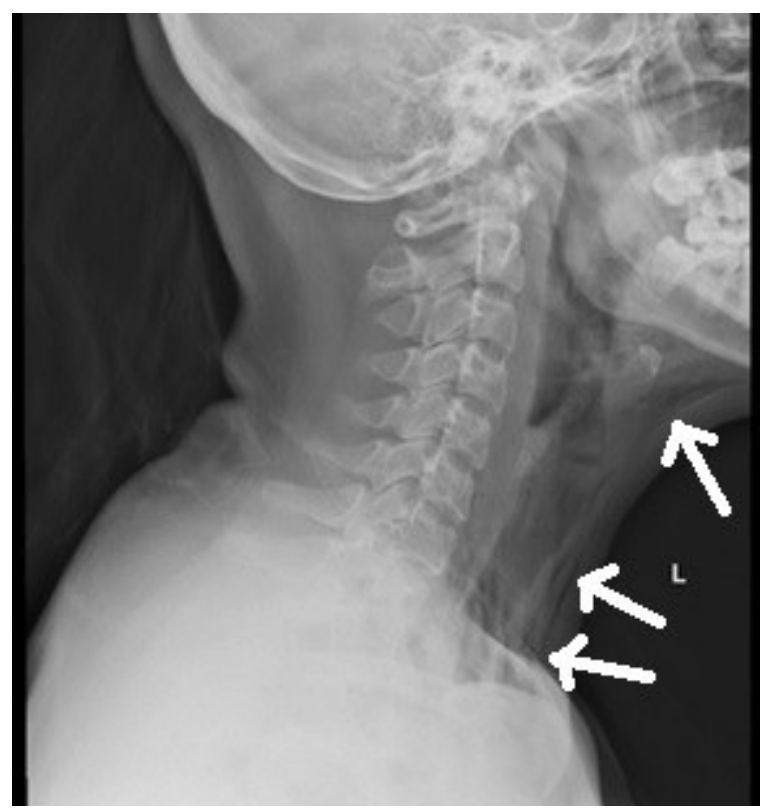

Figure 2: Lateral neck radiograph showed presence of emphysema (indicated by the arrows).

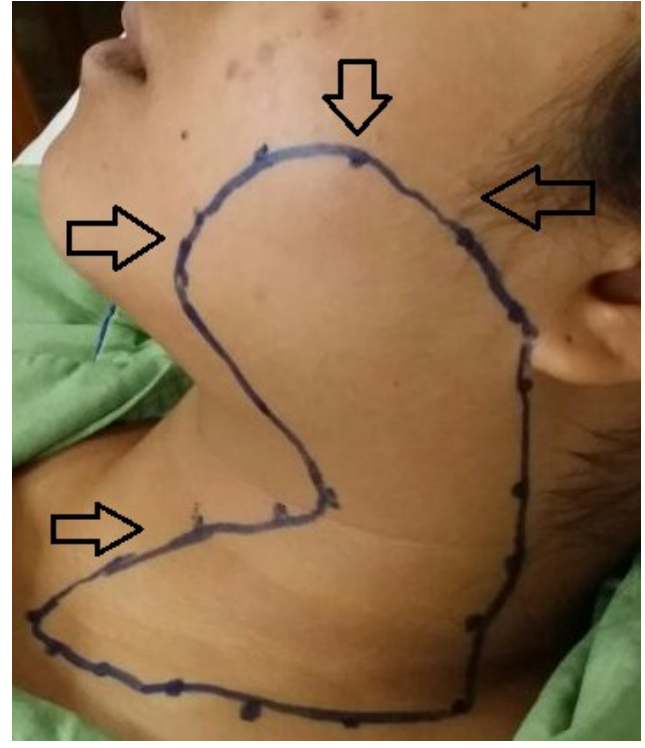

Figure 1B: Subcutaneous emphysema on the left cheek and neck (Lateral view).

emphysema has completely resolved and was discharged home well.

Unfortunately, on the 10th day after surgery, she developed secondary haemmorhage from the left tonsilar bed which required surgical intervention.

The main factor contributing to this complication was poor oral hygiene and the presence of sloughs at the surgical site. She recovered very well.

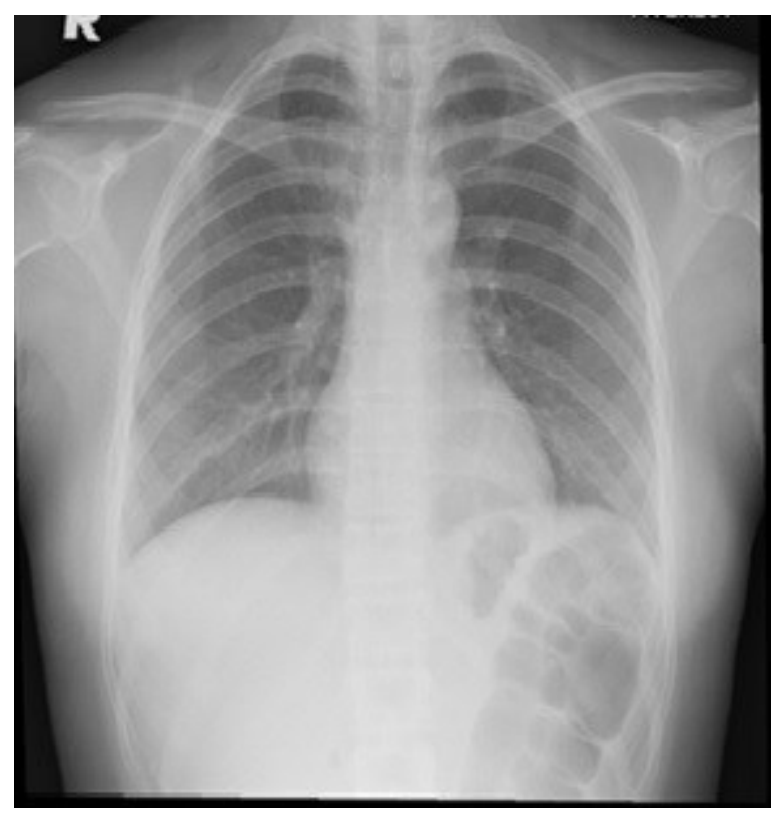

Figure 3: No evidence of pneumothorax and pnuemomediastinum. 


\section{DISCUSSION}

A few possible mechanisms contributing to post tonsillectomy subcutaneous emphysema that has been hypothesized. Air leaking into the subcutaneous plane can be due to overzealous dissection of the tonsils causing injuries to the superior constrictor muscular layer ${ }^{5}$, especially if vasalva manoeuvres are applied. Local anaesthetic infiltration into tonsilar bed may have contributed to this as well ${ }^{6}$. General anaesthesia related causes includes traumatic intubation \& excessive positive pressure ventilation ${ }^{4}$.

Common features of post tonsillectomy subcutaneous emphysema are swelling of face and neck $^{1,2,7,8}$, pressure at facial region ${ }^{1}$, sore throat $^{1}$, increasing odynophagia ${ }^{7}$ and submandibular tenderness ${ }^{3}$.

Besides that, the patient can present with dysphagia, dyspnoea, chest and back pain, cyanosis and Hamman's sign (crunchy sound with each heartbeat) ${ }^{1}$. This is due to the deep dissection of the superior pharyngeal constrictor muscle creates a path through the cervicofacial planes to the parapharyngeal, retropharyngeal and prevertebral spaces. From there, air travelled down to mediastinum through the deep neck spaces and caused pneumomediastinum.

It is important to be aware that this routine procedure can present with rare but possible fatal complications-subcutaneous emphysema and pneumomediastinum. These air collection in the subcutaneous plane can spread to cause upper airway compression and impaired cardiorespiratory functions $\mathrm{s}^{1-3}$. Surgical interventions such as multiple skin punctures and incisions can be performed in the presence of or in anticipation of mild airway compression. In the presence of more severe airway compromise, one patient required tracheostomy ${ }^{9}$ and another patient underwent thoracotomy ${ }^{10}$. Needle thoracostomy is another viable option to resolve cardiorespiratory compromise secondary to pneumomediastinum. Neck exploration surgery can be done to determine and address the source of the subcutaneous emphysema if it is extensive and worsening. Chest tube drainage is done in the presence of pneumothorax secondary to pneumomediastinum. The timing of these procedures depends solely on the severity of airway compromise. No surgical intervention is required if there is no cardiorespiratory compromise. No death related to this complication was ever reported. ${ }^{1}$

Differential diagnosis for facial swelling post tonsillectomy such as hematoma and/or infection must also be ruled out. Most of the reported cases occurred within hours after the surgery ${ }^{4,6,9,10}$, as seen in our surgery. However, there were several reported cases that occurred few days after surgery ${ }^{7}$.

Management of both complications are usually conservative due to its self-limiting nature and resolves spontaneously within 1 week $^{1-10}$ but the duration depends on the severity of the condition. Most importantly is to monitor the progression of the swelling and the marked affected area. Blood investigations that can be done are $\mathrm{FBC}$ and $\mathrm{C}$ Reactive Protein (CRP): to monitor WBC and CRP levels ${ }^{1}$ which can be slightly elevated. Subcutaneous emphysema can be easily detected by cervical and chest radiographs ${ }^{1,3}$. Supplemental oxygen ${ }^{1,3}$, intravenous antibiotics, e.g. cephalosporins ${ }^{1}$ and close monitoring will be sufficient for most cases, although some severe cases might require intensive care management ${ }^{8}$. Only 1 reported case required surgical intervention: tracheostomy to secure the airway ${ }^{1,9}$. Upon discharge, oral antibiotic should be completed for a week duration and the patient is advised to avoid smoking ${ }^{1}$. Activities that can increase upper airway pressure, such as straining, vomiting and coughing should be avoided ${ }^{3}$.

In our case, the anaesthetic procedure and surgery were uneventful. It was an unexpected and rare complication, but we managed to treat her conservatively.

In conclusion, subcutaneous emphysema, and pneumomediastinum of various levels of severity have been known to be a complication for a simple procedure such as tonsillectomy. The most important part is early recognition of symptoms displayed by the patient and to take swift actions. Although tonsillectomy is a minor procedure, patients should be monitored closely during perioperative period and not to be taken lightly because of severe and possibly, fatal complications can occur.

\section{REFERENCES}

1. Bizaki A, Kääriäinen J, Harju T, Rautiainen M. Facial subcutaneous emphysema after tonsillectomy. Head Face Med 2014;10:11.

2. Watanabe K, Kunitomo M, Yamauchi $Y$, et al. Subcutaneous emphysema after tonsillectomy: a case report. J Nippon Med Sch. 2004;71(2):1113.

3. Koukoutsis G, Balatsouras DG, Ganelis P, et al. Subcutaneous Emphysema and 
Pneumomediastinum after Tonsillectomy. Case Rep Otolaryngol 2013; 2013:154857

3. Vos GD, Marres EH, Heineman E, Janssens M. Tension pneumoperitoneum as an early complication after adenotonsillectomy. J Laryngol Otol 1995;109:440-1.

4. Stewart AE, Brewster DF, Bernstein PE. Subcutaneous emphysema and pneumomediastinum complicating tonsillectomy. Arch Otolaryngol Head Neck Surg 2004;130:1324-7.

5. Miman MC, Ozturan O, Durmus M, Kalcioglu MT, Gedik E. Cervical subcutaneous emphysema: an unusual complication of adenotonsillectomy. Paediatr Anaesth 2001;11:491-3.

6. Al Jabr I, Al Harethy S. Cervicofacial subcutaneous emphysema, a rare complication of tonsillectomy. EJENTAS 2014;15(1):49-51.

7. Hung MH, Shih PY, Yang YM, et al. Cervicofacial Subcutaneous Emphysema Following Tonsillectomy: Implications for Anesthesiologists. Acta Anaesthesiologica Taiwanica 2009;47(3):134-7.

8. Panerari AC, Soter AC, Silva FL, et al. Onset of subcutaneous emphysema and pneumomediastinum after tonsillectomy: a case report. Braz J Otorhinolaryngol 2005;71:94-6.

9. Pratt LW, Hornberger HR, Moore VJ. Mediastinal emphysema complicating tonsillectomy and adenoidectomy. Ann Otol Rhinol Laryngol 1962;71:158-69. 